\title{
Linking soil bacterial diversity to ecosystem multifunctionality using backward-elimination boosted trees analysis
}

\author{
Ji-Zheng He $\cdot$ Yuan Ge $\cdot$ Zhihong Xu $\cdot$ Chengrong Chen
}

Received: 6 June 2009 / Accepted: 6 July 2009 /Published online: 31 July 2009

(C) Springer-Verlag 2009

\begin{abstract}
Background, aim, and scope There is increasing evidence of linkages between biodiversity and ecosystem functions. Recent interests on this topic have expanded from an individual-function perspective to a multifunction perspective. This study aims to explore the soil bacterial diversitymultifunctionality relationship.

Materials and methods Soil bacterial diversity was determined using culture-independent molecular techniques. Bacterial taxa groups with positive effects on certain ecosystem functions were identified by aggregated boosted tree analysis with a prediction-error-based backward-elimination criterion. Soil bacterial diversitymultifunctionality relationship was examined by exploring the relationship between the number of ecosystem functions and the number of soil bacterial taxa.

Results More ecosystem functions would require greater numbers of bacterial taxa, which can be explained potentially using the multifunctional complementarity mechanism. Furthermore, a power law equation, $\mathrm{OTU}_{N}=\mathrm{OTU}_{1}(N)^{T}$, was firstly proposed to describe the observed positive relationship between the soil bacterial diversity and the ecosystem
\end{abstract}

Responsible editor: Jianming $\mathrm{Xu}$

Ji-Zheng He and Yuan Ge contributed equally to this work.

J.-Z. He $(\bowtie) \cdot$ Y. Ge

State Key Laboratory of Urban and Regional Ecology,

Research Center for Eco-environmental Sciences,

Chinese Academy of Sciences,

Beijing 100085, China

e-mail: jzhe@rcees.ac.cn

Z. Xu C C. Chen

Center for Forestry and Horticultural Research and School

of Biomolecular and Physical Sciences, Griffith University,

Nathan, Queensland 4111, Australia multifunctionality, where $\mathrm{OTU}_{N}$ is the number of operational taxonomic units (OTUs) required by $N$ functions, while $\mathrm{OTU}_{1}$ is the average number of species required for one function, and $T$ is the average turnover rate of OTU across functions.

Conclusions The number of ecosystem functions would decrease with the loss of soil bacterial diversity. This biodiversity-multifunctionality relationship has an important implication for comprehensively understanding the risk of bacterial diversity loss in relation to the ecosystem functions.

Keywords Bacterial diversity - Belowground ecosystem . Boosted tree analysis · Ecosystem multifunctionality · Microorganisms $\cdot$ Soils

\section{Background, aim, and scope}

The increasing interest in the relationship between biodiversity and ecosystem functions stems from the perceptions that continuous loss of biodiversity, specially caused by human activity, can affect ecosystem functions and services (Loreau et al. 2001; Srivastava and Vellend 2005; Tilman et al. 1997; Worm et al. 2006). However, almost all of the studies in this topic have used an individual-function perspective to examine the relationship between biodiversity and a certain ecosystem function. In fact, most ecosystems are maintained and valued by a series of ecosystem functions and services (Kareiva et al. 2007; Srivastava and Vellend 2005). The use of an individual function as proxy for overall ecosystem functions may ignore other important functions and underestimate the potential risk of biodiversity loss in influencing the overall ecosystem functions (Gamfeldt et al. 2008). Therefore, 
recent interests on this topic start to expand from an individual-function perspective to a multifunction perspective (Gamfeldt et al. 2008; Hector and Bagchi 2007). One recent study has firstly explored the relationship between the grass species diversity and the ecosystem multifunctionality and revealed that more plant species are needed to sustain more ecosystem functions in grassland ecosystems (Hector and Bagchi 2007).

Bacteria are among the most abundant and diverse groups of organisms on earth (Gans et al. 2005) and directly mediate a wide range of ecosystem processes (Balvanera et al. 2006; Bell et al. 2005b; Madsen 2005; Naeem and Li 1997). However, the relationships between bacterial diversity and ecosystem multifunctionality have not been explicitly examined in the belowground ecosystems. Unique aspects of the bacterial biology may prevent them from exhibiting a similar relationship as that found for plant species. For example, bacteria are thought to be flexible in resource requirements and physiological abilities (Prosser et al. 2007) and to obtain new traits easily through horizontal gene transfers that are necessary for survival in a given habitat (Choi and Kim 2007; Thomas and Nielsen 2005). The high degree of ecological redundancy of bacteria can be inferred based on the unique aspects of the bacterial biology (Yin et al. 2000). Then, if bacteria exhibit a high degree of ecological redundancy, the concept that more ecosystem functions require more species to sustain would not be expected. Therefore, we hypothesized that bacterial diversity would not be correlated with the ecosystem multifunctionality.

To examine whether more bacterial taxa are required for more ecosystem functions, we collected soil samples with different management practices from three field sites, including one site at Menangle (AU-MA) in Australia and two sites at Fengqiu (CN-FQ) and Qiyang (CN-QY) in China and examined the differences in soil bacterial communities using culture-independent molecular approaches. The bacterial taxa were defined based on their denaturing gradient gel electrophoresis (DGGE) profiles, and the taxa with positive effects on each function were identified by a backward-elimination aggregated boosted tree (ABT) analysis. A power law equation was proposed to describe the observed relationship and then to predict the least number of bacterial taxa required for a given number of ecosystem functions.

\section{Materials and methods}

\subsection{Site description and sampling}

In total, 53 soil samples were collected from three sampling sites with distinct climatic conditions, soil types, and soil
pH in January and March 2006, to test if the observed soil bacterial diversity-multifunctionality relationships show similar pattern among different sampling sites. In particular, 21 soil samples were collected from AU-MA $\left(34^{\circ} 7^{\prime} \mathrm{S}\right.$, $150^{\circ} 45^{\prime} \mathrm{E}$ ) experimental site of the Center for Recycled Organics in Agriculture in Australia. Sixteen soil samples were collected from CN-FQ $\left(35^{\circ} 00^{\prime} \mathrm{N}, 114^{\circ} 24^{\prime} \mathrm{E}\right)$ and another 16 soil samples from CN-QY $\left(26^{\circ} 45^{\prime} \mathrm{N}\right.$, $110^{\circ} 53^{\prime} \mathrm{E}$ ). $\mathrm{CN}-\mathrm{FQ}$ and $\mathrm{CN}-\mathrm{QY}$ are two experimental stations of Chinese Ecosystem Research Network which was established in 1988 by the Chinese Academy of Sciences as part of a long-term research project to carry out comprehensive ecological monitoring and research on diverse ecosystems. AU-MA had a typical temperate climate with a mean annual rainfall of $615 \mathrm{~mm}$ and a mean annual temperature of $16.3^{\circ} \mathrm{C}$. CN-FQ had a temperate monsoon climate, with a mean annual rainfall of $605 \mathrm{~mm}$ and a mean annual temperature of $13.9^{\circ} \mathrm{C}$. CN-QY had a subtropical monsoon climate, with a mean annual rainfall of $1,288 \mathrm{~mm}$ and a mean annual temperature of $18 \cdot 1^{\circ} \mathrm{C}$. The soil of AU-MA was derived from shale bedrock and classified as hapludalf with silty clay loam texture and $\mathrm{pH}$ ranging from 5.0 to 6.2. The soil of CN-FQ was derived from alluvial sediments of the Yellow River and classified as aquic inceptisols with a sandy loam texture and $\mathrm{pH}$ ranging from 8.4 to 8.8 . The soil of $\mathrm{CN}-\mathrm{QY}$ was derived from quaternary red earth and classified as agri-udic ferrosols with silty clay texture and $\mathrm{pH}$ ranging from 3.9 to 6.0. For the Australian site (AU-MA), the experimental treatments comprised the applications of different quality and quantity of organic manures including Collex Grease Trap Waste, Cleanaway Grease Trap Waste, and Municipal Solid Waste Compost (Ge et al. 2009). For the Chinese sites (CN-FQ and CN-QY), the experimental treatments included the applications of chemical fertilizers $\mathrm{N}, \mathrm{P}, \mathrm{K}$ or in combination with organic manure (He et al. 2007; Ge et al. 2008b). Although the experimental treatments for these three sampling sites were not originally designed to examine the relationship of soil bacterial diversity and ecosystem multifunctionality, the variations in soil bacterial communities and diversity were expected for different soil samples, especially in the condition of long-term treatments of different inorganic or organic fertilizers, which is useful to extract the bacterial taxa groups with specific effects on a certain ecosystem function and then to explore the relationship between the number of functions and the number of bacterial taxa.

Soil samples were collected from experimental plots with different treatments in all the above described Australian and Chinese sites. For each sampling plot, several subsamples of the topsoil were collected and bulked to form one composite sample (about $1 \mathrm{~kg}$ ). Each sample was placed in a sterile plastic bag, sealed, and transported 
to the laboratory on ice. All samples were passed through a $2.0-\mathrm{mm}$ sieve and stored at $4^{\circ} \mathrm{C}$ and $-80^{\circ} \mathrm{C}$, separately. The more detailed information about the sampling regime and soil properties can be found in the previous publications (He et al. 2007; Ge et al. 2008b, 2009).

\subsection{Ecosystem functions}

Ecosystem functions were generally classified as stocks of energy and materials, flows of energy or materials, and the stability of stocks or rates (Srivastava and Vellend 2005). In this work, estimates of both stocks and flows were involved in the analysis. But, for the brief description, we did not differentiate them here. We focused on ecosystem functions specially related to material cycling in the soil ecosystems, which were thought to directly correlate with soil bacteria (He et al. 2006, 2007; Xu and Chen 2006). The ecosystem functions involved in our analysis are soil microbial biomass carbon, organic carbon, nitrate, ammonium, available phosphorous, available potassium, respiration rate, nitrogen mineralization rate, and potential ammonia oxidizing rate.

Microbial biomass carbon was determined by the chloroform fumigation-extraction method (Chen et al. 2004). Soil organic $\mathrm{C}$ was determined by $\mathrm{K}_{2} \mathrm{Cr}_{2} \mathrm{O}_{7}$ oxidation-reduction titration method. The $\mathrm{NH}_{4}{ }^{+}-\mathrm{N}$ and $\mathrm{NO}_{3}{ }^{-}-\mathrm{N}$ were extracted with $2 \mathrm{M} \mathrm{KCl}$ and determined by a continuous-flow analyzer (SAN++, Skalar, Holland). Available phosphorous and available potassium were extracted using $0.5 \mathrm{M} \mathrm{NaHCO}_{3}$ and $1 \mathrm{M}$ ammonium acetate separately and measured using an inductively coupled plasma optical emission spectrometer (Optima 2000DV, Perkin Elmer, USA). Soil respiration rate was determined using the incubation method (Chen et al. 2004). Nitrogen mineralization rate was determined by an anaerobic incubation under laboratory conditions (Chen et al. 2002). Potential ammonia oxidizing rate was measured using a chlorate inhibition method (He et al. 2007).

\subsection{Bacterial community analyses}

Soil DNA was extracted using the Powersoil (Australian samples) and UltraClean (Chinese samples) DNA Isolation Kit (Mo Bio, Carlsbad, USA) according to the manufacturer's instruction. The 16S rRNA gene was PCR-amplified using a protocol described previously (Ge et al. 2008b) with the extracted DNA as template and universal bacterial primers 954F and 1369R ( $\mathrm{Yu}$ and Morrison 2004). Amplicons (about $200 \mathrm{ng}$ ) were analyzed by DGGE using a protocol described previously (Ge et al. 2008a). In brief, the electrophoresis was run for $6 \mathrm{~h}$ at $120 \mathrm{~V}$ and a constant temperature of $60^{\circ} \mathrm{C}$ using $6 \%(w / v)$ acrylamide/bisacrylamide (37.5:1) gels containing a 30-60\% linear gradient of formamide and urea (100\% denaturing solution containing $40 \%[v / v]$ formamide and $7 \mathrm{M}$ urea) in a DCode Universal Mutation Detection System (Bio-Rad Laboratories, Hercules, USA). The gels were stained with 1:10,000 SYBR Gold/Green Nucleic Acid Gel Stain (Invitrogen Molecular Probes, Eugene, USA) for $30 \mathrm{~min}$, scanned by the Gel Documentation System (Bio-Rad Laboratories, Hercules, USA). The bands in DGGE gel were automatically identified by the "detect bands" function in the software Quantity One (Bio-Rad Laboratories, Hercules, USA). Each detected band was defined as an operational taxonomic unit (OTU) according to their relative front values (Bell et al. 2005a; Reche et al. 2005), and then the soil bacterial community profile was reflected by the DGGE fingerprinting pattern. DGGE is a commonly used DNA-based community fingerprinting method for detecting the highthroughput differences between microbial communities (Bell et al. 2005a; Reche et al. 2005). Members of different species may share the same DGGE band (Ge et al. 2008b). Although DGGE patterns provided a different taxonomic resolution than species, it is a consistent measure of community composition (Bell et al. 2005a; Ge et al. 2008a).

2.4 Identifying the OTUs with positive effect on a certain ecosystem function

The OTUs set with positive effect on a certain ecosystem function were identified using a previously described process (Hector and Bagchi 2007) but with some modifications. In brief, the most parsimonious set of OTUs influencing each ecosystem function was firstly identified using prediction error (PE)-based backward-elimination ABT analysis. Then, the OTUs with positive effects on each function were determined from the parsimonious OTUs set by the estimates of the ABT model.

The ABT analysis is a statistical learning method that aims to attain both accurate explanation and prediction for regression and classification analyses (De'ath 2007). The reasons that we employed the $\mathrm{ABT}$ analysis are based on the following: (1) it can increase the accuracy of prediction relative to other methods such as boosted trees, bagged trees, random forests, and generalized additive models (De'ath 2007); and (2) the ABT model can be optimized to achieve a minimally adequate model by comparing PE value, which is well suited for our analytical purpose to determine the parsimonious set of OTUs influencing specific ecosystem function. The PE value of a statistical model is a major estimate to reflect how close the model predictions are to their true values on average. A statistical model with lower PE value means a higher accuracy of the prediction (De'ath 2007).

For PE-based backward-elimination ABT analysis to identify the most parsimonious set of OTUs influencing 
specific ecosystem function, the values of specific ecosystem function parameter (for example, soil microbial biomass) of different soil samples were used as the response variables and the presence/absence values $(1 / 0)$ of different OTUs as the explanatory variables. Before the ABT model fitting, all ecosystem function variables were naturally log-transformed. The PE-based backwardelimination processes are as follows. (1) Starting with a full ABT model involving all OTUs (for example, n OTUs) at a given site, each OTU was eliminated from the original ABT model in turn, and $n$ resulting ABT models were obtained. Each resulting ABT model contains $n-1$ explanatory variables (OTUs). (2) The PE values of the $n$ resulting models were calculated, respectively. (3) By comparison of the PE values of the $n$ resulting models, the OTU whose elimination would cause the greatest decrease of PE value was permanently excluded. These processes (steps 1-3) were repeated until excluding any of the remaining OTUs causing the increase of the $\mathrm{PE}$ value, at which point indicating that a minimally adequate $\mathrm{ABT}$ model had been determined. The OTU assemblage involved in the minimally adequate model was identified as the most parsimonious set of OTUs substantially influencing the ecosystem function in question.

The most parsimonious set of OTUs actually comprises the OTUs with either positive or negative effects on a specific function. The subset of OTUs with positive effects on a specific ecosystem function was selected again from the former parsimonious set of OTUs by the partial dependency plot of the minimally adequate ABT model.

ABT analyses were carried out using the package "gbmplus" (http://www.esapubs.org/Archive/ecol/E088/ 015/suppl-1.htm) within the "R" statistical programming environment (De'ath 2007).

\subsection{Ecosystem multifunctionality analysis}

The relationship between soil bacterial diversity and ecosystem multifunctionality was examined by exploring how the number of bacterial OTUs with positive effects on ecosystem functions changed with the increasing number of ecosystem functions considered. Thus, the numbers of OTUs that had detectable and positive effects on all possible combinations of ecosystem functions at each site were counted according to the results of the ABT analyses. For example, five ecosystem functions investigated at the AU-MA site resulted in 31 counts distributed in five levels of combinations: five at one-function level, ten at two-function level, ten at three-function level, five at four-function level, and one at five-function level.

All relevant statistical analyses were carried out using the package "MASS" (http://cran.r-project.org/web/packages/ $\mathrm{VR} /$ index.html) within the "R" statistical programming environment (Venables and Ripley 2002).

\section{Results}

The numbers of the OTUs of the bacterial communities with positive effects on different ecosystem functions were four (microbial biomass carbon), seven (ammonium), seven (soil respiration rate), eight (nitrate), and 11 (nitrogen mineralization rate) at the AU-MA site; four (soil organic carbon), five (nitrate), eight (microbial biomass carbon), eight (ammonium), eight (available potassium), and nine (available phosphorous) at the CN-FQ site; and six (soil organic carbon), eight (microbial biomass carbon), 11 (nitrate), and 16 (potential ammonia oxidizing rate) at the CN-QY site, respectively. Although they showed some differences between the functions and between the sites, chi-squared test revealed that there were no conventionally significant differences between the functions (Poisson analysis of deviance: $\chi_{8}^{2}=12.25, P=0.14$ ) and between the sites (Poisson analysis of deviance: $\left.\chi_{2}^{2}=3.85, P=0.15\right)$.

The relationship between number of OTUs and number of functions at each site is plotted in Fig. 1 and showed a significantly positive but slightly asymptotic relationship. To quantitatively describe this relationship, a conceptual equation was proposed to generalize the empirical relationships as a power law:

$\mathrm{OTU}_{N}=\mathrm{OTU}_{n}\left(\frac{N}{n}\right)^{T}$

where both $N$ and $n$ are the numbers of functions involved in analysis; $\mathrm{OTU}_{N}$ is the number of OTUs required by $N$ functions; $\mathrm{OTU}_{n}$ is the number of OTUs required by $n$ functions which are part of $N$ functions, and $T$ is the turnover rate of OTU when the considered number of functions increased from $n$ to $N$. Here, different OTUs in specific OTU set were assumed functionally equivalent. When $n$ equals to 1, Eq. 1 can be simplified to:

$\mathrm{OTU}_{N}=\mathrm{OTU}_{1}(N)^{T}$

Parameters in Eq. 2 have explicit ecological meanings, where $\mathrm{OTU}_{1}$ is the average number of bacterial OTU required for one function, and $T$ is a measure of the average turnover rate of OTU across functions. Therefore, the average number of OTUs required for one function and the average turnover rate of OTU across functions can be estimated by the parameter evaluation to Eq. 2 using the observed data.

The fitted relationships based on our data were $\mathrm{OTU}_{N}=7.3 N^{0.84}\left(R^{2}=0.80, P<0.0001\right)$ at the AU-MA site, $\mathrm{OTU}_{N}=7.1 N^{0.71}\left(R^{2}=0.80, P<0.0001\right)$ at the CN-FQ site, and $O T U_{\mathrm{N}}=10.0 N^{0.80}\left(R^{2}=0.69, P<0.001\right)$ at the CN-QY site (see Fig. 1). $R^{2}$ was calculated based on Pearson product-moment correlation coefficient. Therefore, the estimated average numbers of OTUs for one function were 

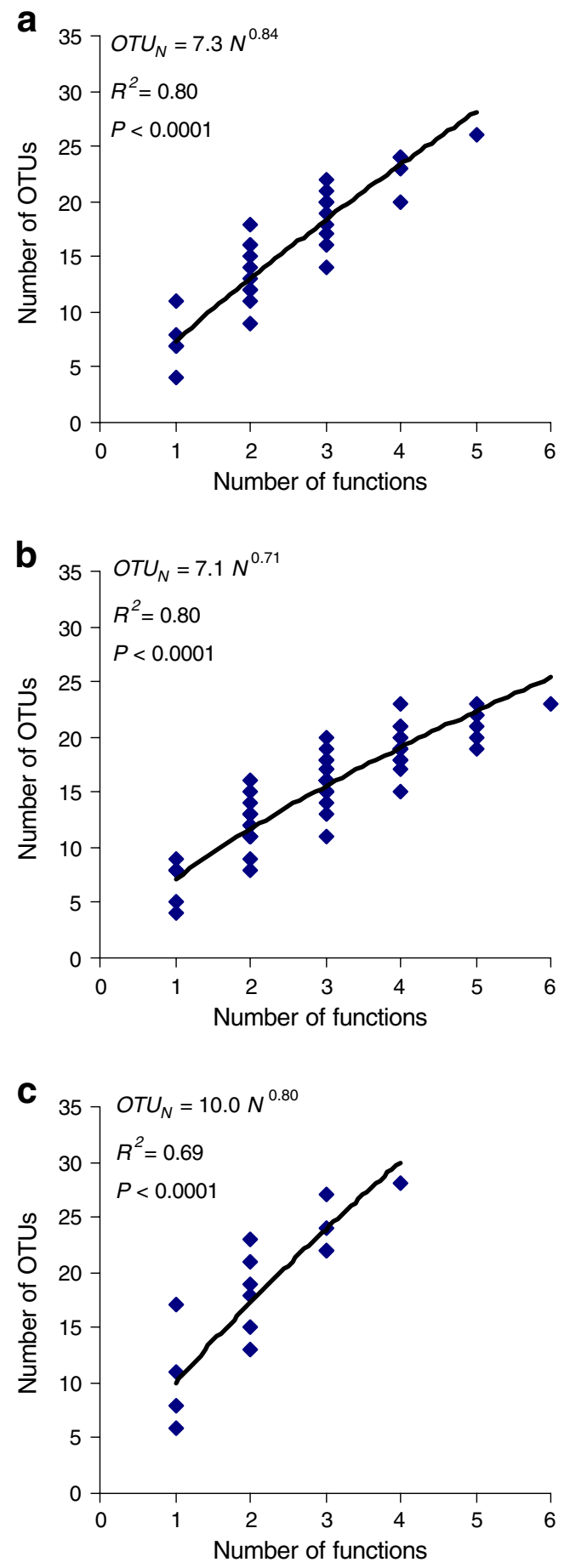

Fig. 1 The relationship between the number of ecosystem functions involved in the analysis and the number of OTUs with positive effects on functions. More bacterial diversity (the number of OTUs derived from DGGE, $O T U_{N}$ ) is required for sustaining more ecosystem functions (the number of ecosystem functions involved in the analysis, $N$ ) according to the power law $O T U_{N}=7.3 N^{0.84}\left(R^{2}=0.80, P<0.0001\right)$ at the AU-MA site (a), $O T U_{N}=7.1 N^{0.7 .1}\left(R^{2}=0.80, P<0.0001\right)$ at the CN-FQ site (b), and $O T U_{N}=10.0 N^{0.80}\left(R^{2}=0.69, P<0.001\right)$ at the CN-QY site (c). AU, Australia; CN, China; DGGE, denaturing gradient gel electrophoresis; FQ, Fengqiu; MA, Menangle; OTU, operational taxonomic unit; QY, Qiyang
$7.3,7.1$, and 10.0 at these three sampling sites, respectively, and the number of required OTUs changed with turnover rates of $0.84,0.71$, and 0.80 across the functions at the three corresponding sampling sites (Table 1).

\section{Discussion}

\subsection{Bacterial diversity-multifunction relationship}

Our results explicitly showed that, despite the unique aspects of bacterial biology, greater bacterial diversity was required for sustaining more ecosystem functions. The observed positive relationship between the needed number of bacterial OTUs and the number of functions considered could be used to reject the null hypothesis that a certain group of bacteria could contribute to all ecosystem functions in the soil ecosystem and then to support the hypothesis that, like most relationships between biodiversity and the strength of single ecosystem function (Balvanera et al. 2006; Naeem and Li 1997; Naeem et al. 1994; Srivastava and Vellend 2005), there is a certain correlation between the bacterial diversity and the number of ecosystem functions. Similarly, a recent study has examined the relationship between plant diversity and ecosystem multifunctionality in grassland ecosystems using an Akaike Information Criterion based backward-deletion regression technique and shown that more ecosystem functions do require more grass diversity (Hector and Bagchi 2007). Another study has shown that the probability of sustaining multiple functions increases with more species included, using a 0.5 threshold for evaluating if a single ecosystem function was performed sufficiently according to some monoculture datasets (Gamfeldt et al. 2008). All of these studies, including our work here, indicate that the positive relationship between biodiversity and ecosystem multifunctionality could be a common scenario, which

Table 1 Summary of bacterial multiple functions analysis

\begin{tabular}{lcccrc}
\hline Site & $\begin{array}{l}\text { Number } \\
\text { of } \\
\text { functions }\end{array}$ & $\begin{array}{l}\text { Number } \\
\text { of OTU } \\
\text { pool }\end{array}$ & $\begin{array}{l}\text { Number of } \\
\text { OTUs for all } \\
\text { functions }\end{array}$ & OTU $_{1}$ & $T$ \\
\hline AU-MA & 5 & 57 & 26 & 7.3 & 0.84 \\
CN-FQ & 6 & 49 & 23 & 7.1 & 0.71 \\
CN-QY & 4 & 64 & 28 & 10.0 & 0.80
\end{tabular}

Number of functions the number of ecosystem functions investigated per site, Number of OTU pool the total number of OTUs present differences between samples per site, Number of OTUs for all functions the cumulative number of OTUs with positive effects on at least one ecosystem function, $O T U_{1}$ the estimated average number of OTUs required for one ecosystem function, $T$ the estimated average turnover rate of OTU across functions, $A U$ Australia, $C N$ China, $F Q$ Fengqiu, $M A$ Menangle, $O T U$ operational taxonomic unit, $Q Y$ Qiyang 
could apply to both microorganism communities and macroorganism communities. Furthermore, unlike other studies using manipulated communities which included small number of selected species, we examined the relationship between the bacterial diversity and multiple functions in soil ecosystems using the whole bacteria communities which included a very large number of species featured by high-throughput molecular biological approaches.

The power law equation, $\mathrm{OTU}_{N}=\mathrm{OTU}_{1}(N)^{T}$, was firstly proposed to quantitatively describe the biodiversity-multifunctionality relationship. The reasons why we use a power law equation to describe the biodiversity-multifunctionality relationship are due to the fact that this equation is easy to understand and the parameters in this equation have explicit ecological meanings. For example, $\mathrm{OTU}_{1}$ is the average number of OTUs required for one function, and $T$ is the average turnover rate of OTU across the functions. However, it needs to be noted that the proposed power law equation is just a conceptual model based on the assumption that different OTUs with positive effect on specific ecosystem function are functionally equivalent. Furthermore, the functional parameters measured in this study can be affected not only by bacteria but also by other biological groups. Therefore, better assessments of ecosystem multifunctionality could be made by considering multiple trophic levels simultaneously (Hector and Bagchi 2007). Although the power law equation needs to be further validated from more studies in different ecosystems and for different organisms, the observed data in our study evidently demonstrated its good fitness at all sampling sites across the Northern and Southern hemispheres with distinct climatic conditions, soil types, and soil $\mathrm{pH}$. Therefore, this type of biodiversity-multifunctionality relationship described by the power law equation could be quite universal across different sampling locations.

Despite the potentially general power law form, the parameters of $\mathrm{OTU}_{1}$ and $T$ showed some differences among our three sampling sites. The variation of OTU $\mathrm{O}_{1}$ between the sites probably arises from a mixture of biological and functional differences. For example, they may partly reflect the variation in the size of the OTU pools at the different sites, as that with the higher $\mathrm{OTU}_{1}$ was also that with higher OTU pool (Hector and Bagchi 2007). They also may partly reflect the variation in the ecosystem functions involved in the analysis, as the number of OTUs with positive effect on each function was different for different functions.

Variation in the $T$ values is of interest because it indicates that different behaviors underlie the biodiversity-multifunctionality relationship at different multifunction scales. This can be illustrated by considering two extreme situations. In one extreme scenario, if each group of bacterial taxa (it is still possible that the bacterial taxa group included just one OTU) is completely complementary to different functions, effects of different group of bacterial taxa are additive, and a positive linear relationship will be observed between the number of OTUs and the number of functions. In this situation, $T$ value should be 1 and the power law equation transfers to a linear equation, $\mathrm{OTU}_{N}=\mathrm{OTU}_{1} N$. On the other hand, if a single subset of bacterial taxa in the taxa pool contributes to all the functions (it seems unlikely that a single taxa could control all ecosystem functions), then the remaining bacterial taxa are functionally redundant and have no effects on sustaining more ecosystem functions. In that situation, $T$ value should be 0 , and the power law equation becomes $\mathrm{OTU}_{N}=\mathrm{OTU}_{1}$, where $\mathrm{OTU}_{1}$ indicates the size of the single group of bacterial taxa that affects all ecosystem functions. In most natural situations, species seems, to some extent, to be functionally complementary and redundant, and $T$ value should be between 0 and 1 , which is as observed in our study.

As the slope of the biodiversity-multifunctionality curve, $T$ reflects the rate at which OTU richness increases with the number of functions involved in the analysis. A higher rate of biodiversity-multifunctionality between the samples implies a faster turnover in bacterial taxa assemblage across functions and hence a steeper relationship. It should be noted that parameter $T$ in the power law equation is an overall measure of the turnover rate of OTU assemblage across the functions. The bacterial $T$ value may also be functionally dependent. That is to say, the $T$ value may vary with the variation of the number of functions involved in the analysis. For example, for the Australian site, the $T$ value of bacterial biodiversity-multifunctionality curve was generally increased when the number of functions involved in the analysis decreased from five to four.

It is important to note that the estimated bacterial biodiversity-multifunctionality relationship may depend on the defined taxa. The DGGE-defined OTU used in this study was a different taxonomic resolution to the species. The estimate of $\mathrm{OTU}_{1}$ based on the DGGE fingerprinting method may underestimate the true number of bacterial species (Muyzer et al. 1993) with positive effects on one function in our samples. However, the estimated slope of bacterial diversity-multifunctionality curve, $T$, seems to be stable if different bacterial taxa definitions result in common proportional changes in species richness among the samples, especially for those samples collected from a similar habitat (Bell et al. 2005a).

\subsection{Implications of the biodiversity-multifunctionality} relationship

The positive biodiversity-multifunctionality relationship has important implications for understanding the potential 
mechanisms underlying the relationship between bacterial diversity and ecosystem multifunctionality. For the relationship of biodiversity and individual ecosystem function, most studies have revealed a generally positive but asymptotic relationship (Srivastava and Vellend 2005). The positive relationship suggests the functional complementarity among some species (Loreau 1998; Loreau and Hector 2001; Tilman et al. 1997) or the positive selection effect (Cardinale et al. 2006; Loreau and Hector 2001; Tilman et al. 1997) with increasing biodiversity, which are thought to be the two principal mechanisms to understand how biodiversity affects a certain individual ecosystem function (Loreau et al. 2001). About the relationship between biodiversity and ecosystem multifunctionality (the number of functions), a similar mechanism of multifunctional complementarity can be inferred to explain the observed positive relationship. Niche differentiation between species or genotypes allows them to use different resources. Diversity-rich communities can therefore contribute to more ecosystem functions, especially those functions related to material cycling.

Our results, combined with other studies (Gamfeldt et al. 2008; Hector and Bagchi 2007), have a fundamental implication for comprehensively understanding the influence of biodiversity loss on the ecosystem functions. These results suggest that the loss of biodiversity can influence the ecosystem, not only the degree of a single function but also the number of functions. Therefore, studies focusing on the influence of biodiversity loss on single ecosystem function may underestimate the risk of biodiversity loss (Hector and Bagchi 2007). Furthermore, the decelerating decrease of the number of ecosystem functions with the loss of soil bacterial diversity was observed in this study as shown by the positive but asymptotic biodiversity-multifunctionality curve. This result suggests that the loss of biodiversity will influence the ecosystem multifunctionality faster at the beginning of the biodiversity loss than that at the following stage of the biodiversity loss. Therefore, when the biodiversity of ecosystem decreases from the original stage to the depauperate stage, for example, because of the increasing human disturbance, the relatively minor decrease of the biodiversity starting from the full diversity stage will cause the loss of certain ecosystem functions. Our results further highlight the significance of biodiversity conservation by indicating the conditions under which high-risk consequence of biodiversity loss can occur.

\section{Conclusions}

To our knowledge, this paper explores for the first time the bacterial diversity-multifunctionality relationship in natural soil ecosystems using combined molecular techniques and advanced statistical analyses. Our results have clearly shown that, similar to that found for plant species, more ecosystem functions do require greater numbers of bacterial taxa, which offer the exciting potential for a more synthesized view for both microorganism and macroorganism biodiversity. Furthermore, a power law equation was proposed for the first time to describe the observed positive relationship between soil bacterial diversity and ecosystem multifunctionality. These results have an important implication for comprehensively understanding the risk of bacterial diversity loss in relation to the ecosystem functions.

Acknowledgements This research was jointly supported by the Chinese Academy of Sciences (KZCX2-YW-408, KZCX1-YW-0603), the Natural Science Foundation of China (40871129), and the Australian Research Council. The assistances of the staff at the Australian and Chinese sites for soil sampling and data collecting are sincerely appreciated.

\section{References}

Balvanera P, Pfisterer AB, Buchmann N, He J-S, Nakashizuka T, Raffaelli D, Schmid B (2006) Quantifying the evidence for biodiversity effects on ecosystem functioning and services. Ecol Lett 9:1146-1156

Bell T, Ager D, Song J-I, Newman JA, Thompson IP, Lilley AK, van der Gast CJ (2005a) Larger islands house more bacterial taxa. Science 308:1884

Bell T, Newman JA, Silverman BW, Turner SL, Lilley AK (2005b) The contribution of species richness and composition to bacterial services. Nature 436:1157-1160

Cardinale BJ, Srivastava DS, Duffy JE, Wright JP, Downing AL, Sankaran M, Jouseau C (2006) Effects of biodiversity on the functioning of trophic groups and ecosystems. Nature 443:989-992

Chen CR, Xu ZH, Hughes J (2002) Effects of nitrogen fertilization on soil nitrogen pools and microbial properties in a hoop pine (Araucaria cunninghamii) plantation in southeast Queensland, Australia. Biol Fert Soils 36:276-283

Chen CR, Xu ZH, Mathers NJ (2004) Soil carbon pools in adjacent natural and plantation forests of subtropical Australia. Soil Sci Soc Am J 68:282-291

Choi I-G, Kim S-H (2007) Global extent of horizontal gene transfer. Proc Natl Acad Sci USA 104:4489-4494

De'ath G (2007) Boosted trees for ecological modeling and prediction. Ecology 88:243-251

Gamfeldt L, Hillebrand H, Jonsson PR (2008) Multiple functions increase the importance of biodiversity for overall ecosystem functioning. Ecology 89:1223-1231

Gans J, Wolinsky M, Dunbar J (2005) Computational improvements reveal great bacterial diversity and high metal toxicity in soil. Science 309:1387-1390

Ge Y, He J-Z, Zhu Y-G, Zhang J-B, Xu ZH, Zhang L-M, Zheng Y-M (2008a) Differences in soil bacterial diversity: driven by contemporary disturbances or historical contingencies? ISME J 2:254-264

Ge Y, Zhang J-B, Zhang L-M, Yang M, He J-Z (2008b) Long-term fertilization regimes affect bacterial community structure and diversity of an agricultural soil in northern China. J Soils Sediment 8:43-50 
Ge Y, Chen CR, Xu ZH, Eldridge SM, Chan KY, He Y, He J-Z (2009) Carbon/nitrogen ratio as a major factor for predicting the effects of organic wastes on soil bacterial communities assessed by DNA-based molecular techniques. Environ Sci Pollut Res . doi:10.1007/s11356-009-0185-6

He J-Z, Xu ZH, Hughes J (2006) Molecular bacterial diversity of a forest soil under residue management regimes in subtropical Australia. Fems Microbiol Ecol 55:38-47

He J-Z, Shen J-P, Zhang L-M, Zhu Y-G, Zheng Y-M, Xu M-G, Di H-J (2007) Quantitative analyses of the abundance and composition of ammonia-oxidizing bacteria and ammonia-oxidizing archaea of a Chinese upland red soil under long-term fertilization practices. Environ Microbiol 9:2364-2374

Hector A, Bagchi R (2007) Biodiversity and ecosystem multifunctionality. Nature 448:188-190

Kareiva P, Watts S, McDonald R, Boucher T (2007) Domesticated nature: shaping landscapes and ecosystems for human welfare. Science 316:1866-1869

Loreau M (1998) Biodiversity and ecosystem functioning: a mechanistic model. Proc Natl Acad Sci USA 95:5632-5636

Loreau M, Hector A (2001) Partitioning selection and complementarity in biodiversity experiments. Nature 412:72-76

Loreau M, Naeem S, Inchausti P, Bengtsson J, Grime JP, Hector A, Hooper DU, Huston MA, Raffaelli D, Schmid B, Tilman D, Wardle DA (2001) Biodiversity and ecosystem functioning: current knowledge and future challenges. Science 294:804-808

Madsen EL (2005) Identifying microorganisms responsible for ecologically significant biogeochemical processes. Nat Rev Microbiol 3:439-446

Muyzer G, de Waal E, Uitterlinden A (1993) Profiling of complex microbial populations by denaturing gradient gel electrophoresis analysis of polymerase chain reaction-amplified genes coding for 16S rRNA. Appl Environ Microbiol 59:695-700

Naeem S, Li S (1997) Biodiversity enhances ecosystem reliability. Nature 390:507-509
Naeem S, Thompson LJ, Lawler SP, Lawton JH, Woodfin RM (1994) Declining biodiversity can alter the performance of ecosystems. Nature 368:734-737

Prosser JI, Bohannan BJM, Curtis TP, Ellis RJ, Firestone MK, Freckleton RP, Green JL, Green LE, Killham K, Lennon JJ, Osborn AM, Solan M, van der Gast CJ, Young JPW (2007) The role of ecological theory in microbial ecology. Nat Rev Microbiol 5:384-392

Reche I, Pulido-Villena E, Morales-Baquero R, Casamayor EO (2005) Does ecosystem size determine aquatic bacterial richness? Ecology 86:1715-1722

Srivastava DS, Vellend M (2005) Biodiversity-ecosystem function research: is it relevant to conservation? Annu Rev Ecol Evol S $36: 267-294$

Thomas CM, Nielsen KM (2005) Mechanisms of, and barriers to, horizontal gene transfer between bacteria. Nat Rev Microbiol 3:711-721

Tilman D, Lehman CL, Thomson KT (1997) Plant diversity and ecosystem productivity: theoretical considerations. Proc Natl Acad Sci USA 94:1857-1861

Venables WN, Ripley BD (2002) Modern applied statistics with S. Springer, Berlin

Worm B, Barbier EB, Beaumont N, Duffy JE, Folke C, Halpern BS, Jackson JBC, Lotze HK, Micheli F, Palumbi SR, Sala E, Selkoe KA, Stachowicz JJ, Watson R (2006) Impacts of biodiversity loss on ocean ecosystem services. Science 314:787-790

$\mathrm{Xu} \mathrm{ZH}$, Chen CR (2006) Fingerprinting global climate change and forest management within rhizosphere carbon and nutrient cycling processes. Environ Sci Pollut Res 13:293-298

Yin B, Crowley D, Sparovek G, De Melo WJ, Borneman J (2000) Bacterial functional redundancy along a soil reclamation gradient. Appl Environ Microbiol 66:4361-4365

Yu Z, Morrison M (2004) Comparisons of different hypervariable regions of $r r s$ genes for use in fingerprinting of microbial communities by PCR-denaturing gradient gel electrophoresis. Appl Environ Microbiol 70:4800-4806 